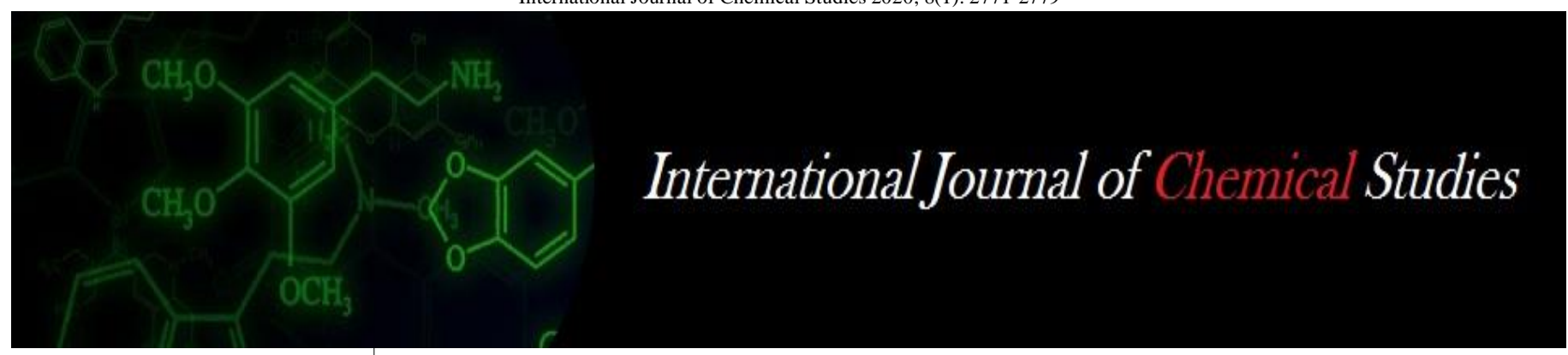

P-ISSN: 2349-8528

E-ISSN: 2321-4902

www.chemijournal.com

IJCS 2020; 8(1): 2771-2779

(C) 2020 IJCS

Received: 16-11-2019

Accepted: 20-12-2019

\section{Kumari Rekha}

Department of Plant Breeding and Genetics, Bihar Agricultural University, Sabour, Bhagalpur, Bihar, India

Ravi S Singh

Department of Plant Breeding and Genetics, Bihar Agricultural University, Sabour, Bhagalpur, Bihar, India

Anjani Kumar

Department of Plant Breeding and Genetics, Bihar Agricultural University, Sabour, Bhagalpur, Bihar, India

Dharamsheela Thakur

Department of Molecular

Biology and Genetic

Engineering, Bihar Agricultural

University, Sabour, Bhagalpur,

Bihar, India

\section{Chandan Kishore}

Department of Plant Breeding and Genetics, Bihar Agricultural University, Sabour, Bhagalpur, Bihar, India

\section{Sima Sinha}

Department of Plant Breeding and Genetics, Bihar Agricultural University, Sabour, Bhagalpur, Bihar, India

\section{Ankita Sinha}

Department of Plant Breeding and Genetics, Bihar Agricultural University, Sabour, Bhagalpur, Bihar, India

\section{Prabhash K Singh}

Department of Plant Breeding and Genetics, Bihar Agricultural University, Sabour, Bhagalpur, Bihar, India

Corresponding Author: Ravi S Singh

Department of Plant Breeding and Genetics, Bihar Agricultural University, Sabour, Bhagalpur, Bihar, India

\section{RAPD and ISSR derived SCAR marker(s) for Aphid tolerance in Brassica juncea Czern. And Coss}

\author{
Kumari Rekha, Ravi S Singh, Anjani Kumar, Dharamsheela Thakur, \\ Chandan Kishore, Sima Sinha, Ankita Sinha and Prabhash K Singh
}

DOI: https://doi.org/10.22271/chemi.2020.v8.i1ap.8689

\begin{abstract}
In this present study, eight genotypes of Brassica juncea comprising four tolerant (IC-399802, IC491089, IC-312545, IC-312553) and four susceptible (IC-385686, IC-264131, IC-426392, Laxmi) genotypes for aphid tolerance were used to generate $\underline{\text { Sequence }}$ Characterized Amplified Region (SCAR) markers through dominant PCR based markers (51 RAPD and 12 ISSR markers). 13 RAPD (Random Amplified Polymorphic DNA) and 8 ISSR (Inter Simple Sequence Repeat) markers were found to be polymorphic but only 3 primers OPE16 (RAPD), UBC 839 (ISSR) and UBC 864 (ISSR) were obtained which could distinguish tolerant genotypes from susceptible ones. OPE16 (RAPD) obtained unique band of size approx. $600 \mathrm{bp}$ in susceptible genotypes while it was absent in the tolerant genotypes. Similarly, UBC 839 (ISSR) yielded $~ 800$ bp unique band in bulk tolerant while UBC 864 (ISSR) yielded three bands of $\sim 1200 \mathrm{bp}, \sim 1000 \mathrm{bp}$, and $\sim 500 \mathrm{bp}$ in tolerant genotypes which was absent in the susceptible genotypes. These unique bands were excised to generate five sets of SCAR markers. Among the five sets of SCAR marker, only BJSCAR F1 and BJSCAR R1 set yielded the promising result in all for 4 susceptible genotypes as well as bulk susceptible and was absent in all the tolerant genotypes and Brassica fruticulosa (highly tolerant to aphid, used as a control). So, this SCAR marker developed could be successfully used in screening of B.juncea genotypes in future breeding programs.
\end{abstract}

Keywords: Brassica juncea, mustard aphid, RAPD, ISSR, SCAR marker

\section{Introduction}

Brassica juncea (L.) Czern. \& Coss. Commonly known as 'Indian Mustard' is one of the highly important crop species from the family Brassicaceae. It is a Rabi season crop and highly demanded as edible oil. But, one of its major biotic constraints is mustard aphid (Lipaphis erysimi K.) which hampers its productivity. It belongs to order Homoptera and family aphidae. Both adults and nymphs stages adversely affects the plant growth and development by sucking the saps of the leaf, inflorescence and pods of the plant rendering weak and fragile plants. According to some reports, L.erysimi can cause 10-90\% loss in yield in rapeseed-mustard. Though, mustard aphids can be controlled satisfactorily by insecticides, but the residual effect of the toxic chemicals hampers the environment as well as the friendly insects. So, development of resistant/tolerant varieties is the best approach to tackle the menance of aphid.

Apart from development of resistant/tolerant varieties, it is further important to develop reliable screening techniques. In B. juncea, some of the morphological and biochemical traits like small and hardy inflorescence with loosely packed buds, darker leaves, more branches with wider angle of orientation, less amount of total sugar and sulphur contents, higher glucosinolates particularly sinigrin traits were observed to be related to aphid tolerance (Rai \& Sehgal, Ahuja et al., Martínez-Ballesta et al. $)^{[13,1,7]}$. Till date there is no report of successful tolerant cultivars developed by conventional means in $B$. juncea with systemic plant responses in the form of direct or indirect defenses against aphid attack. At the same time, a suitable high-throughput method for screening large numbers of genotypes yet to be developed in breeding for selection of tolerant cultivars in B. juncea. Prior reports suggest efforts were made in many crops to study the resistance to biotic stresses using molecular markers (Myburg et al. Prabhu et al. Monika et al. Chander et al. $)^{[10,12,9,2]}$. 
However, limited information is available in $B$. juncea related to development of SCAR markers for aphid resistance, which are not good enough for high level of confirmation (Chander et al.) ${ }^{[2]}$.

Sequence Characterized Amplified Region (SCAR) markers are important codominant molecular markers used for tagging of a gene or to link with a specific trait. Therefore, it would be useful to develop a good SCAR marker related to aphid tolerance for screening of $B$. juncea genotypes.

\section{Material and Method}

\subsection{Plant material}

The plant materials included four tolerant and four susceptible genotypes of $B$. juncea identified on the basis of earlier field trials conducted at Oilseed section of PBG Department, BAC, Sabour.

\subsection{DNA extraction}

Total DNA was extracted from leaves collected in the field following the CTAB method described by Doyle and Doyle ${ }^{[5]}$ with a few modifications using $100 \mathrm{mg}$ of leaf tissue in liquid nitrogen using mortar and pestle. For bulked DNA analysis, two DNA bulks were constructed, each using the four genotypes with either tolerance or susceptibility for the aphid infestation. Later, the integrity of DNA was checked by gel electrophoresis on $0.8 \%$ Agarose-EtBr gel. Gel was viewed on a UV trans-illuminator and captured on gel documentation system (UVITEC, Cambridge, U.K.).

\subsection{PCR setup}

The PCR was carried out on a thermal cycler (Veriti R\#9902, ABI, Singapore) as follows for $15 \mu \mathrm{l}$ PCR reaction: $7.5 \mu \mathrm{l}$ of 2X Primix Taq (Xcelris Genomics, India), $0.5 \mu 1$ of primer $(10 \mu \mathrm{M}), 5 \mu \mathrm{l}$ of distilled autoclaved water was added and further $2 \mu \mathrm{l}$ of DNA (50 ng) was added. The reaction was carried out in thermal cycler, initial denaturation at $94^{\circ} \mathrm{c}$ for 5 min, 40 cycles of denaturation at $94^{\circ} \mathrm{C}$ for $1 \mathrm{~min}$, annealing at $32-49{ }^{\circ} \mathrm{C}$ for $1 \mathrm{~min}$, extension at $72{ }^{\circ} \mathrm{C}$ for $1 \mathrm{~min}$, and final extension at $72^{\circ} \mathrm{C}$ for $7 \mathrm{~min}$ with the list of the primers as mentioned in the table 1.0. The PCR product was resolved by gel electrophoresis on $1.5 \%$ Agarose-EtBr gel. Gel was viewed on a UV trans-illuminator and captured on gel documentation system (UVITEC, Cambridge, U.K.).

\subsection{Band excision and elution}

The six unique bands selected were cut and eluted using Quick gel extraction method as per the protocol of manufactures (Thermo-fisher, USA).

\subsection{Ligation reaction for TA cloning}

For the ligation reaction following constituents as per the manufacturer's instruction (Thermo-fisher, USA) were used

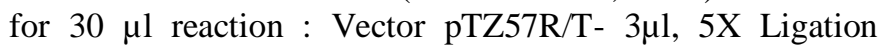
buffer - $6 \mu \mathrm{l}, \mathrm{T}_{4}$ ligase- $1 \mu \mathrm{l}$, DNA $-10 \mu \mathrm{l}$ and autoclaved distill water $-10 \mu \mathrm{l}$ and was incubated for 1 hour at $22^{\circ} \mathrm{C}$. Chilled on ice and transform $10 \mu \mathrm{l}$ of reaction into $50 \mu \mathrm{l}$ competent cells.

\subsection{Transformation in competent cells of DH5 $\alpha$ strains of} E. coli

DH5 $\alpha$ E. coli cells (NEB, U.K.) were transformed with the ligated product using the standard protocol (Sambrook et al.) ${ }^{[14]}$. After an hour these cells were spread on Luria-Bertini (LB) agar plates containing ampicillin $(100 \mu \mathrm{l} / \mathrm{ml})$ and X-gal $(20 \mu \mathrm{l} / \mathrm{ml})$.

\subsection{Re-streaking of colony}

All the white (transformed) colonies were re-streaked on a fresh ampicllin plate containing ampicillin $(100 \mu \mathrm{l} / \mathrm{ml})$ and $\mathrm{X}$ gal $(20 \mu \mathrm{l} / \mathrm{ml})$ and incubated for overnight at $37^{\circ} \mathrm{C}$.

\subsection{Confirmation of cloning of desired bands}

The cloning of desired bands were confirmed by double digestion with EcoRI and BamHI and colony PCR using vector specific M13 forward and reverse primers.

\subsubsection{Double digestion using restriction enzymes}

For the confirmation of insert restriction enzyme based double digestion was performed using following constituents for 30 $\mu \mathrm{l}$ reaction: $10 \mu \mathrm{l}$ Plasmid, $0.5 \mu \mathrm{l}$ restriction enzyme BamH1and EcoR1 (10U/ $\mu$ l each, Thermo Scientific, USA ) each was used, $2 \mu$ l Tango buffer and $17 \mu$ l autoclaved distilled water was added to make up the volume. The setup was incubated for 1 hour and checked on 1\% agarose gel and the gel was viewed on a UV trans- illuminator and captured on gel documentation system (UVITEC, Cambridge, UK).

\subsubsection{Colony PCR}

Colony PCR was performed to check the cloning of PCR product. Plasmid containing the ligated DNA insert was confirmed by colony PCR using vector specific primer pairs flanking the cloning site $\{\mathrm{M} 13$ forward (F) 5'GTAAAACGACGGCCAGTG-3' and M13 reverse (R) 5'GGAAACAGCTATGACCATG-3'\}. For colony lysate

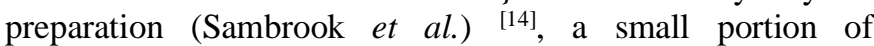
transformed bacterial colony was picked up with a clean micro-tip and transferred into $50.0 \mu \mathrm{l}$ of colony lysis buffer. The micro-centrifuge tubes were incubated in a boiling water bath for $10 \mathrm{~min}$ and chilled on ice for $2 \mathrm{~min}$. After cooling, cell debris was pelleted by centrifugation for $2 \mathrm{~min}$ and the supernatant (colony lysate) was transferred to a new microcentrifuge tube. PCR reaction was set-up as follows $7.5 \mu \mathrm{l}$ of 2X Premix Taq (Xcelris Genomics, India), $0.5 \mu 1$ of each M13 $\mathrm{F}$ and M13 R primer $(10 \mu \mathrm{M}), 5 \mu \mathrm{l}$ of distilled autoclaved water was added and further $2 \mu \mathrm{l}$ of DNA (50 ng) was added. The PCR parameters were: 25 cycles of $94{ }^{\circ} \mathrm{C}$ for $30 \mathrm{sec}, 54$ ${ }^{\circ} \mathrm{C}$ for $40 \mathrm{sec}, 72{ }^{\circ} \mathrm{C}$ for $1-3 \mathrm{~min}$ and final extension at $72{ }^{\circ} \mathrm{C}$ for $5 \mathrm{~min}$, and cooling to $4{ }^{\circ} \mathrm{C}$. PCR amplified products were electrophoresed on $1.2 \%$ agarose $+\mathrm{EtBr}$ gel for analysis.

\subsection{Plasmid Extraction}

Plasmid from transformed clones was extracted following method (Sambrook et al. $)^{[14]}$.

\subsection{Sequencing}

Plasmids were sequenced at Xcelris Genomics, Ahmadabad using both M13 forward and M13 reverse primers for forward and reverse sequencing, respectively.

\subsection{Blast analysis}

The nucleotide sequence obtained was screened for vector sequence contamination initially through online VecScreen (https://www.ncbi.nlm.nih.gov/tools/vecscreen/), and then manually looked at vector's flanking sequence near multiple cloning sites (MCS). Then BLASTN analysis (http://blast.ncbi.nlm.nih.gov/Blast.cgi) was done to find if any similarity exists in GenBank database.

\subsection{Primer designing}

Then the actual sequence was used for SCAR primer 
designing by online programme Primer 3 Input (http://bioinfo.ut.ee/primer3-0.4.0/).

\subsection{Validation of Primer}

SCAR primers developed were validated by PCR using DNA samples from four aphid susceptible genotypes (1) Laxmi (2) IC-426392 (3) IC-399802 (4) IC-264131 (5) bulk of all four susceptible and four aphid tolerant genotypes (6) IC-385686 (7) IC-312553 (8) IC-491089 (9) IC-312545 and (10) bulk of all four tolerant along with (11) B. fruiticulosa as control for aphid tolerant.

\section{Result}

DNA fingerprinting of four susceptible and four tolerant genotypes was carried out using fifty-one RAPD primers and twelve ISSR primers. Out of fifty-one RAPD primers and twelve ISSR primers, only thirteen RAPD primers and eight ISSR primers showed amplifications. Among thirteen RAPD primers and eight ISSR primers, only one RAPD primer OPE 16 and two ISSR primer UBC 839 and UBC 864 produced five unique bands which discriminated tolerant genotypes from susceptible ones.

In RAPD primer OPE 16 produced one bands of size $\sim 600 \mathrm{bp}$ (Fig. 1.1.) which could discriminate tolerant genotypes from susceptible ones for aphid tolerance. Similarly, UBC 839 yielded $\sim 800$ bp unique band in bulk tolerant (Fig.1.2.) while UBC 864 yielded three bands of $\sim 1200 \mathrm{bp}, \sim 1000 \mathrm{bp}$, and $\sim 500 \mathrm{bp}$ in tolerant genotypes (Fig. 1.3.) respectively.

These unique bands were excised and eluted from the gel. Further, ligated into TA-cloning vector (Invitrogen, ThermoFischer, U.S.A.) and transformed into E.coli (DH5a) cells (NEB, U.K.) following a standard transformation protocol (Sambrook et al.) ${ }^{[14]}$. Blue- white screening method was performed for the selection of white positive clones as shown in the Fig 1.4.

Further the insert of the clone was confirmed by double digestion using EcoRI and Bam HI and colony PCR. The representative figure of double digestion and Colony PCR is shown in Fig. 1.5(a.) and Fig. 1.5 (b.), respectively.

\subsection{Sequencing and analysis of cloned RAPD/ISSR fragments}

The sequencing of plasmids was performed by Sanger's dideoxy method with M13F and M13 R primers for forward and reverse sequencing, respectively. These sequences were first looked for cloning site specific sequences and for primer sequences. Vector sequence contamination was also checked by VecScreen online tool(https://www.ncbi.nlm.nih.gov/tools/vecscreen/). Thus, the resulting sequences of RAPD/ISSR fragments i.e. SCAR sequences named as BJSCAR1, BJSCAR2, BJSCAR3, BJSCAR4 and BJSCAR5. These sequences were searched for similarity in GenBank nucleotide database using BLASTN programme (https://blast.ncbi.nlm.nih.gov) and BJSCAR2, BJSCAR3, BJSCAR4 and BJSCAR5 showed similarity with genomic sequence of Raphanus sativus, Brassica napus, Arabidopsis thaliana except BJSCAR 1.

\subsection{Studying polymorphism through developed SCAR markers}

The sequences obtained were used to design seven sets of SCAR primers using online tool available at http://bioinfo.ut.ee/primer3-0.4.0. Out of seven sets of SCAR primers designed from unique band sequences, BJSCAR1-F1 and BJSCAR1-R1 (Table 2.0) yielded a prominent unique bands in all the four susceptible genotypes as well as in the bulk susceptible and was absent in the tolerant genotypes under study (Fig.1.6). This primer set also did not show any amplification in $B$. fruticulosa, a highly tolerant to aphid (used as control), thereby confirms this SCAR primer set's discriminatory power for aphid susceptible and tolerant genotypes of B. juncea (Fig. 1.6.). Other SCAR primers (BJSCAR1-F2 and BJSCAR1-R2 for SCAR1; BJSCAR2-F1 and BJSCAR2-R1 for SCAR2; BJSCAR3-F1, BJSCAR3-R1, BJSCAR3-F2 and BJSCAR-R2 for SCAR3; BJSCAR4-F1, BJSCAR4-R1 for SCAR4; BJSCAR5-F1, BJSCAR5-R1 for SCAR5) could not show clear cut polymorphism between susceptible and tolerant genotypes (Fig. 1.7-1.10).

\section{Discussion \\ 4.1 Conversion of RAPD/ISSR-derived fragments into SCAR marker}

In the present study, RAPD/ISSR-derived fragments were cloned, sequenced and converted into SCAR markers namely, BJSCAR1, BJSCAR2, BJSCAR3, BJSCAR4 and BJSCAR5. BLASTN analysis of these SCAR sequences was done to see if any of these related to resistance. The sequences of BJSCAR2, BJSCAR3, BJSCAR4, BJSCAR5 showed high similarity with nucleotide sequences of A. thaliana, B. rapa, B. napus and other Brassica spp. but none specifically related to resistance. BJSCAR1 did not show any match in GenBank nucleotide database, this could be a novel sequence.

There were several reports on RAPD/ISSR-derived SCAR markers, developed for polymorphism study related to biotic and abiotic stresses in crops ${ }^{[3,4,15]}$. A list of important traits linked with molecular markers in B. juncea is shown in Table 2.3. SCAE1 and SCAE2 primers were designed that discriminated heat tolerant and susceptible tomato ${ }^{[3]}$. In sugarcane, RAPD- derived SCAR marker (OPAK 12724) developed was used for screening tolerant and susceptible genotypes to drought stress [15]. Similarly, for powdery mildew in pea, a RAPD-derived SCAR marker ScOPX 04880 was developed to screen for the resistance gene 'erl' in tolerant and susceptible genotypes ${ }^{[16]}$. In B. juncea, putative source of aphid resistance reported was based on the molecular analysis of the identified tolerant accessions to mustard aphid [2]. They screened 34 germplasm with 284 RAPD primers, of which 87 primers showed amplification, and from these four were polymorphic and finally one RAPD primer could clearly discriminated the tolerant and susceptible accessions, converted SCAR marker ${ }^{[2]}$.

\subsection{SCAR primer developed to discriminate the aphid} susceptible and tolerant genotypes of $B$. juncea

Out of seven sets of SCAR primers obtained from SCAR marker sequences, BJSCAR1-F1 and BJSCAR1-R1 yielded a prominent unique bands in all the four susceptible genotypes as well as in the bulk susceptible and was absent in the tolerant genotypes (Fig.1.6). This primer set also did not show any amplification in $B$. fruticulosa, a highly tolerant to aphid (used as control), thereby confirms this SCAR primer set's discriminatory power for aphid susceptible and tolerant genotypes of mustard. Other SCAR primers (BJSCAR2-F1 and BJSCAR2-R1 for SCAR2; BJSCAR3-F1, BJSCAR3-R1 for SCAR3; BJSCAR4-F1, BJSCAR4-R1 for SCAR4; BJSCAR5-F1, BJSCAR5-R1 for SCAR5) could not show clear cut polymorphism between susceptible and tolerant genotypes (Fig. 4.7.2- Fig. 4.7.5). This indicates that probably polymorphism of RAPD/ISSR markers from which these markers were derived lost upon conversion into SCAR 
marker. There are reports that conversion of RAPD to SCAR markers resulted in a loss of polymorphism linked to tolerance-sensitivity to Fusicoccum in almond ${ }^{[8]}$, this loss of polymorphism has also been reported elsewhere ${ }^{[6,17]}$. Even in case of SCAR marker reported in B. juncea for aphid resistance could not provide unequivocal results since the association between marker and resistance was not always unidirectional and suggested for refinement of the marker ${ }^{2}$. SCAR markers also reported to yield ambiguous results or polymorphism and the reason assigned was possibility of either original RAPD polymorphisms was caused by mismatches in nucleotides in the priming sites or due to the crossing over between the gene controlling the trait and marker ${ }^{11}$. Hence, our emphasis should be on confirming reproducibility of results using these markers for a given trait or characteristics. SCAR marker developed in the present study could be further refined using more aphid resistant genotypes for its wider applicability in Brassica spp.

Thus, in the present study, only one SCAR marker was developed and validated in different susceptible/tolerant genotypes of $B$. juncea. This marker distinguished susceptible and tolerant genotypes. This was also tested with $B$. fruiticulosa, an aphid tolerant genotypes, which further confirms its discriminatory power. We further suggests that it could be further refined using more aphid resistant genotypes for its wider applicability in Brassica spp.

Table 1: List of RAPD/ISSR primers used in the present study

\begin{tabular}{|c|c|c|c|}
\hline S. No & Primer Name & Sequence $\left(5^{\prime}\right.$ to $\left.3^{\prime}\right)$ & $\operatorname{Tm}\left({ }^{0} \mathrm{C}\right)$ \\
\hline 1. & OPA06 & 5' GGTCCCTGAC & 34 \\
\hline 2. & OPA08 & 5' GTGACGTAGG & 32 \\
\hline 3. & OPA09 & 5' GGGTAACGCC & 34 \\
\hline 4. & OPA12 & $5^{\prime}$ TCGGCGATAG & 32 \\
\hline 5. & OPA13 & 5' CAGCACCCAC & 34 \\
\hline 6. & OPA14 & 5' TCTGTGCTGG & 32 \\
\hline 7. & OPA15 & 5' TTCCGAACCC & 32 \\
\hline 8. & OPA17 & 5' GACCGCTTGT & 32 \\
\hline 9. & OPC01 & 5' TTCGAGCCAG & 32 \\
\hline 10 & OPC05 & 5' GATGACCGCC & 34 \\
\hline 11. & OPC06 & 5' GAACGGACTC & 32 \\
\hline 12. & OPC07 & 5' GTCCCGACGA & 34 \\
\hline 13. & OPC09 & 5' CTCACCGTCC & 34 \\
\hline 14. & OPC17 & 5' TTCCCCCCAG & 34 \\
\hline 15. & OPC18 & $5^{\prime}$ TGAGTGGGTG & 32 \\
\hline 16. & OPE01 & 5' CCCAAGGTGC & 34 \\
\hline 17. & OPE02 & 5' GGTGCGGGAA & 34 \\
\hline 18. & OPE04 & 5' GTGACATGCC & 32 \\
\hline 19. & OPE11 & 5' GAGTCTCAGG & 32 \\
\hline 20. & OPE14 & $5^{\prime}$ TGCGGCTGAG & 34 \\
\hline 21. & OPE16 & 5' GGTGACTGTG & 32 \\
\hline 22. & OPE17 & 5' CTACTGCCGT & 32 \\
\hline 23. & OPE18 & 5' GGACTGCAGA & 32 \\
\hline 24. & OPE19 & $5^{\prime}$ ACGGCGTATG & 32 \\
\hline 25. & OPE20 & 5' AACGGTGACC & 32 \\
\hline 26. & OPH01 & 5' GGTCGGAGAA & 32 \\
\hline 27. & OPH02 & 5' TCGGACGTGA & 32 \\
\hline 28. & $\mathrm{OPH} 03$ & 5' AGACGTCCAC & 32 \\
\hline 29. & OPH04 & 5' GGAAGTCGCC & 34 \\
\hline 30. & OPH09 & 5' TGTAGCTGGG & 32 \\
\hline 31. & OPH13 & 5' GACGCCACAC & 34 \\
\hline 32. & Rapid1 & 5' CGTACTGCAG & 32 \\
\hline 33. & Rapid2 & 5' CGTCACAATG & 32 \\
\hline 34. & Rapid3 & 5'GGTGCGAGCT & 32 \\
\hline 35. & Rapid4 & 5'CTCTGACGGC & 34 \\
\hline 36. & Rapid5 & 5'GGATTACGTG & 32 \\
\hline 37. & Rapid6 & 5'CACCGAAACA & 34 \\
\hline 38. & Rapid7 & 5'GGCACCGTCA & 32 \\
\hline 39. & Rapid8 & 5' CATGGCACTG & 32 \\
\hline 40. & Rapid9 & 5'TCCACACAGA & 32 \\
\hline 41. & Rapid10 & 5' GCCGACGATG & 34 \\
\hline 42. & Rapid11 & 5'TGTTTGCGCC & 32 \\
\hline 43. & Rapid12 & 5'AAGCTAGCCC & 32 \\
\hline 44. & Rapid13 & 5' GGGCCCACAC & 34 \\
\hline 45. & Rapid14 & 5' TGCCGAGACG & 32 \\
\hline 46. & Rapid15 & 5'TGCCGAGACG & 34 \\
\hline 47. & Rapid16 & 5' CAGAAGTGGG & 32 \\
\hline 48. & Rapid17 & 5' CGTGGACACT & 32 \\
\hline 49. & Rapid18 & 5' GTTTAGCGCA & 32 \\
\hline 50. & Rapid19 & 5'AAACGGTTCG & 34 \\
\hline 51 & Rapid20 & 5' AGTCCAGCTA & 32 \\
\hline 52. & UBC807 & 5' AGAGAGAGAGAGAGAGT & 45 \\
\hline
\end{tabular}




\begin{tabular}{|c|c|c|c|}
\hline 53. & UBC808 & 5' AGAGAGAGAGAGAGAGC & 47 \\
\hline 54. & UBC816 & 5' CACACACACACACACAT & 46 \\
\hline 55. & UBC827 & 5' ACACACACACACACACG & 46 \\
\hline 56. & UBC832 & 5' ACACACACACACACACYC & 49 \\
\hline 57. & UBC839 & 5' CACACACACACACACARG & 49 \\
\hline 58. & UBC840 & 5' CTCTCTCTCTCTCTCTRA & 48 \\
\hline 59. & UBC843 & 5' CTCTCTCTCTCTCTCTRC & 48 \\
\hline 60. & UBC844 & 5' ACACACACACACACACYG & 48 \\
\hline 61. & UBC857 & 5'ATGATGATGATAGATGATG & 48 \\
\hline 62. & UBC864 & 5' GACAGACAGACAGACA & 43 \\
\hline 63. & UBC873 & & 43 \\
\hline
\end{tabular}

Table 2: List of SCAR primers developed

\begin{tabular}{|c|c|c|c|c|c|}
\hline S. No. & Name & Sequencest $\left(5^{\prime} 3^{\prime}\right)$ & No. of bases & $\operatorname{Tm}\left({ }^{\circ} \mathrm{C}\right)$ & Amplicon Size (bp) \\
\hline 1. & BJSCAR1-F1 & CAGAAAAATGTGACCCTGAC & 20 & 56 & \multirow{2}{*}{193} \\
\hline 2. & BJSCAR1-R1 & TCACAACACTGGTGTCAATC & 20 & 55 & \\
\hline 3. & BJSCAR1-F2 & TTAACCAGACCGTTGATTCT & 20 & 55 & \multirow{2}{*}{221} \\
\hline 4. & BJSCAR1-R2 & TGTCAGGGTCACATTTTTCT & 20 & 55 & \\
\hline 5. & BJSCAR2-F1 & ATGCATTGTTATCTCCACCT & 20 & 55 & \multirow{2}{*}{219} \\
\hline 6. & BJSCAR2-R1 & TCTTCATCCTCGTTGTCTTT & 20 & 55 & \\
\hline 7. & BJSCAR3-F1 & TTCAGAGTAGTTGCTTGTCTCC & 22 & 56 & \multirow{2}{*}{157} \\
\hline 8. & BJSCAR3-R1 & GCTCCATATCTTGTTCTCTGTT & 22 & 55 & \\
\hline 9. & BJSCAR3-F2 & GAGAGAGTACACCTTTTGTGGT & 22 & 55 & \multirow{2}{*}{217} \\
\hline 10. & BJSCAR3-R2 & СТTCССТTTCTCAGCTCTCT & 20 & 55 & \\
\hline 11. & BJSCAR4-F1 & GATGCATTGTTATCTCCACCT & 21 & 57 & \multirow{2}{*}{153} \\
\hline 12. & BJSCAR4-R1 & GCTTACTTGGCTTTAGTGTTTC & 22 & 55 & \\
\hline 13. & BJSCAR5-F1 & GATGATGCATTGTTATCTCCAC & 22 & 57 & \multirow{2}{*}{156} \\
\hline 14. & BJSCAR5-R1 & GCTTACTTGGCTTTAGTGTTTC & 22 & 55 & \\
\hline
\end{tabular}

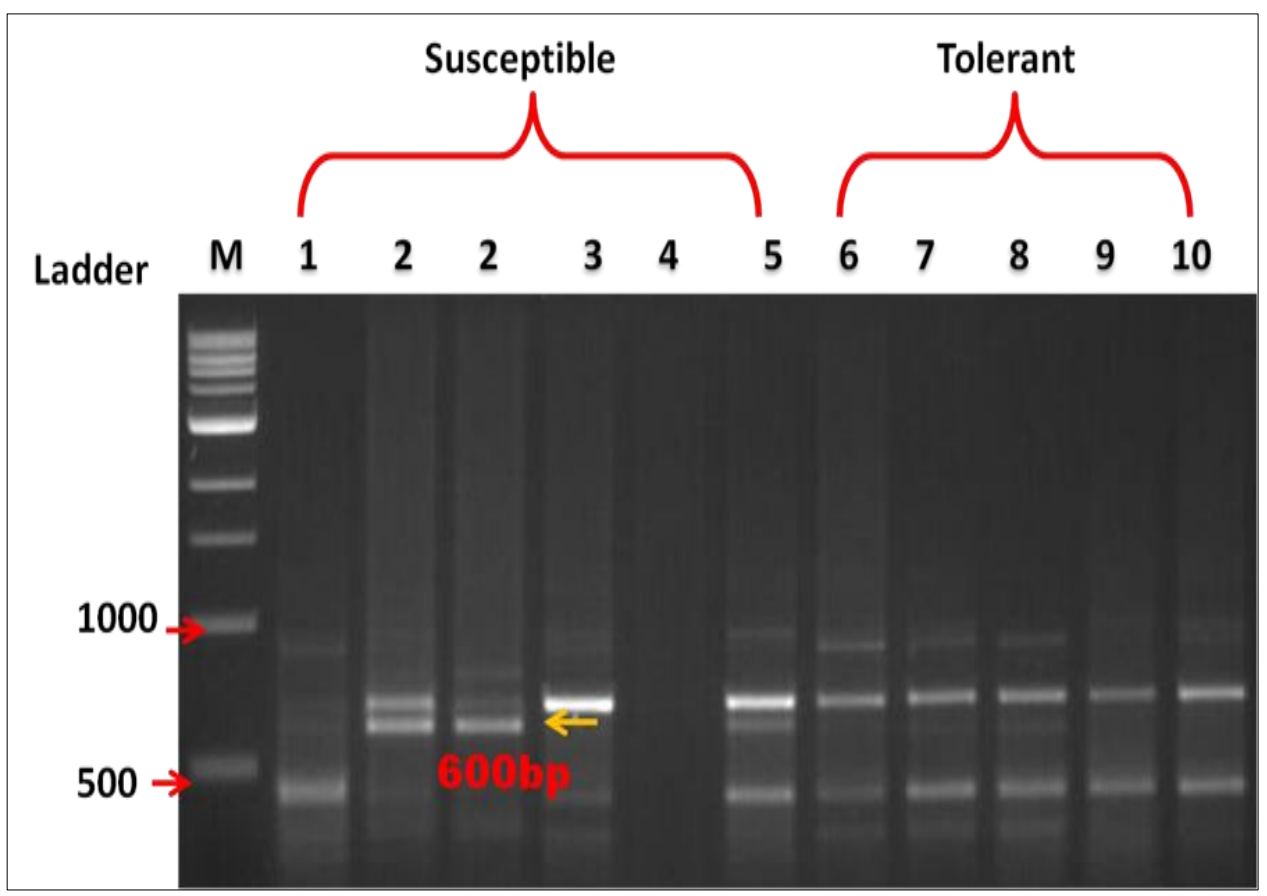

Fig 1.1: RAPD banding pattern of tolerant and sensitive cultivars obtained with the RAPD primer OPE16. Where, 1-4 represents Susceptible genotypes namely 1. Laxmi, 2. IC 426392, 3. IC 399802, 4. IC 264131, and 5. Bulk of these susceptible genotypes (1-4); 6-9 represents Tolerant genotypes namely 6. IC 385686, 7. IC 312553, 8. IC 491089, 9. IC 312545, and 10. Bulk of these tolerant genotypes (6-9); M indicates 500 bp DNA ladder. Band excised for cloning is indicated with yellow arrow. 


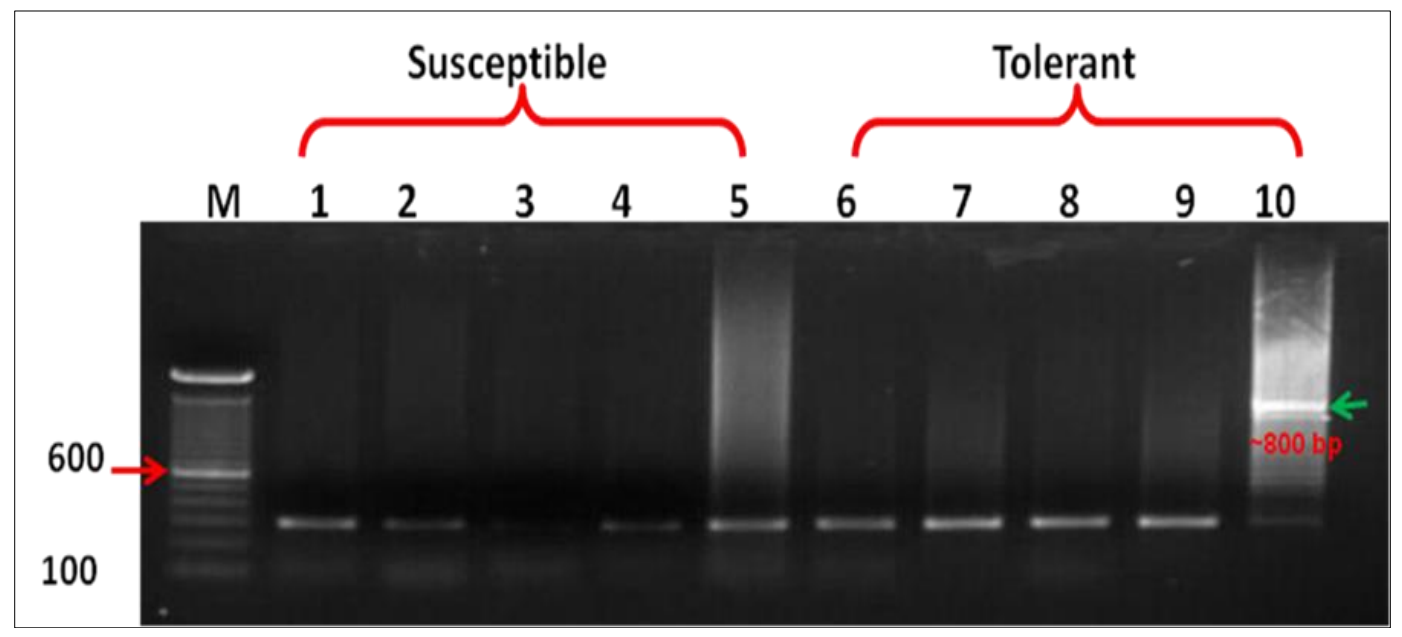

Fig 1.2: DNA banding pattern of tolerant and sensitive cultivars obtained with the ISSR primer UBC 839. Where, 1-4 represents Susceptible genotypes namely 1. Laxmi, 2. IC 426392, 3. IC 399802, 4. IC 264131, and 5. Bulk of these susceptible genotypes (1-4); 6-9 represents Tolerant genotypes namely 6. IC 385686, 7. IC 312553, 8. IC 491089, 9. IC 312545, and 10. Bulk of these tolerant genotypes (6-9); M indicates 100 bp DNA ladder. Band excised for cloning is indicated with green arrow

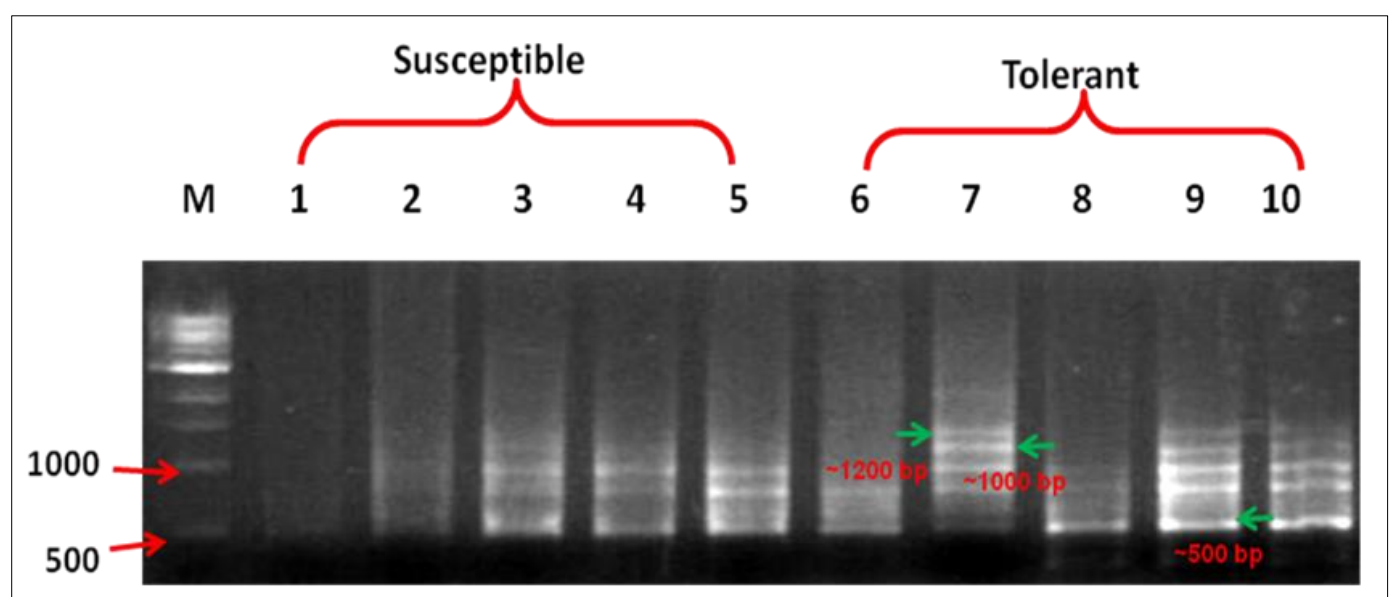

Fig 1.3: DNA banding pattern of tolerant and sensitive cultivars obtained with the ISSR primer UBC 864. Where, 1-4 represents Susceptible genotypes namely 1. Laxmi, 2. IC 426392, 3. IC 399802, 4. IC 264131, and 5. Bulk of these susceptible genotypes (1-4); 6-9 represents Tolerant genotypes namely 6. IC 385686, 7. IC 312553, 8. IC 491089, 9. IC 312545, and 10. Bulk of these tolerant genotypes (6-9); M indicates 500 bp DNA ladder. Band excised for cloning is indicated with green arrow
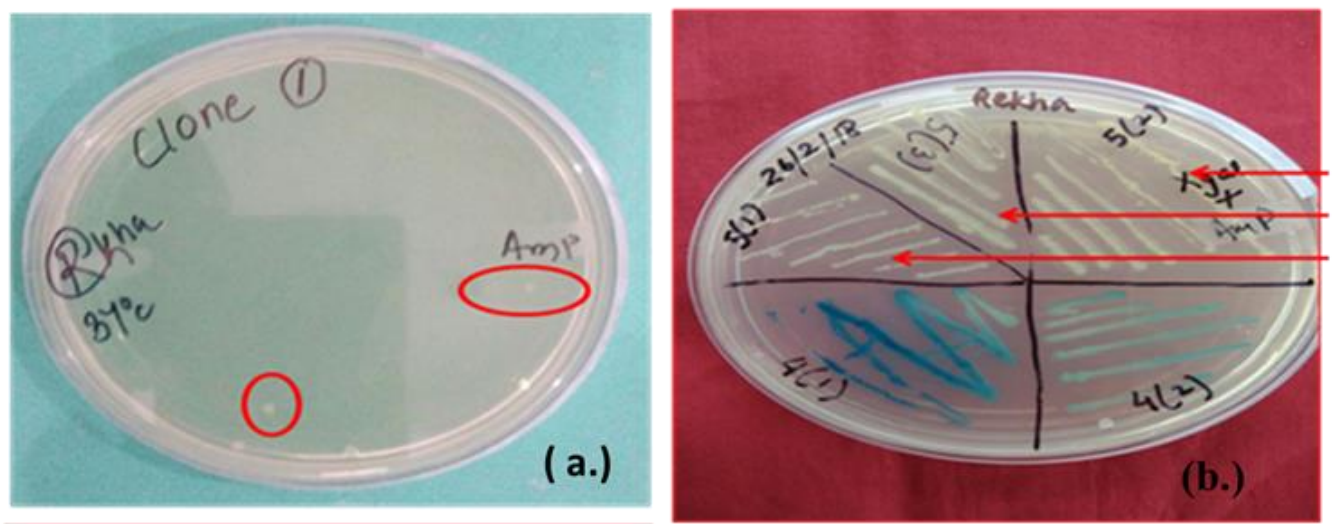

\section{Positive clones}

Fig 1.4: Screening of DH5 $\alpha$. coli cells transformed with fragments ligated into pTZ57R/T cloning vector, on LB-Amp agarplates at $37^{\circ} \mathrm{C}(\mathrm{a})$, Blue-white screening on X-gal-Amp-LB agar plates (b). 

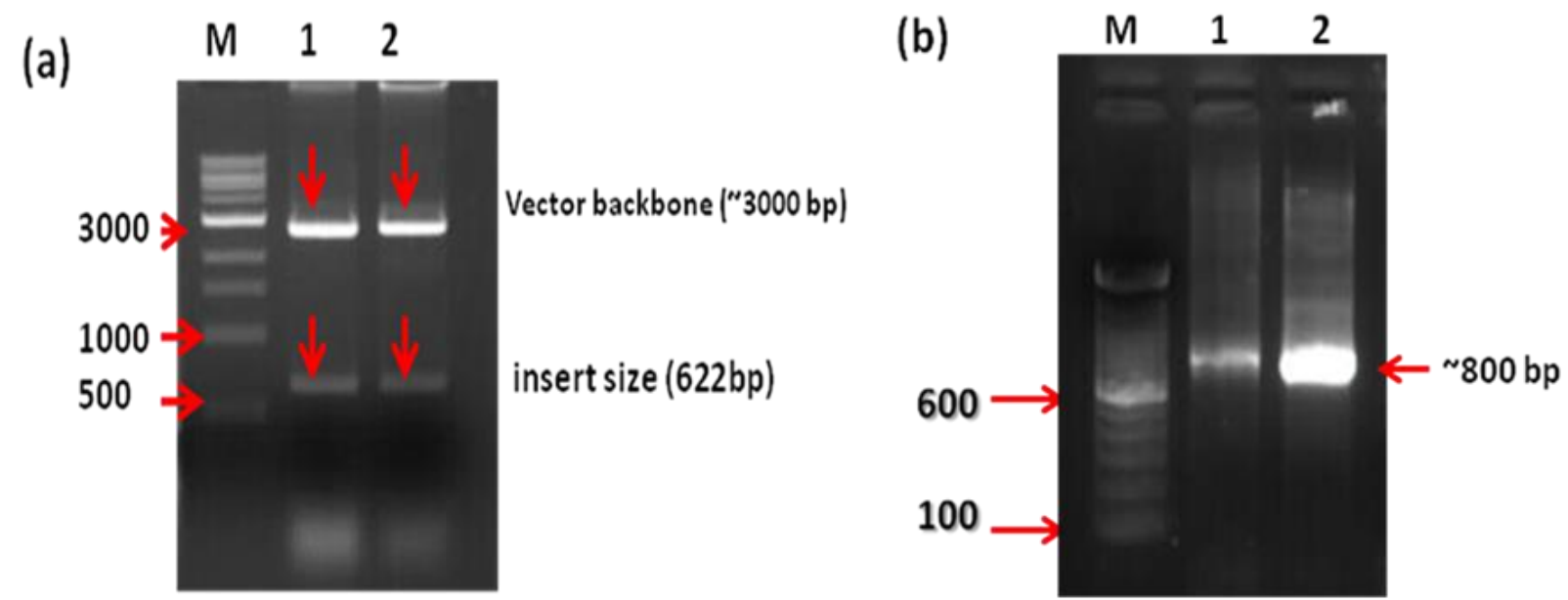

Fig 1.5: Confirmation of inserts by Double digestion with EcoR1 and Bam HI (a), and by Colony PCR using M13 F and M13 R primers (amplicon size of $~ 800$ bp includes size of insert plus distance between M13F \& R binding sites) (b). M, 100 bp DNA ladder.

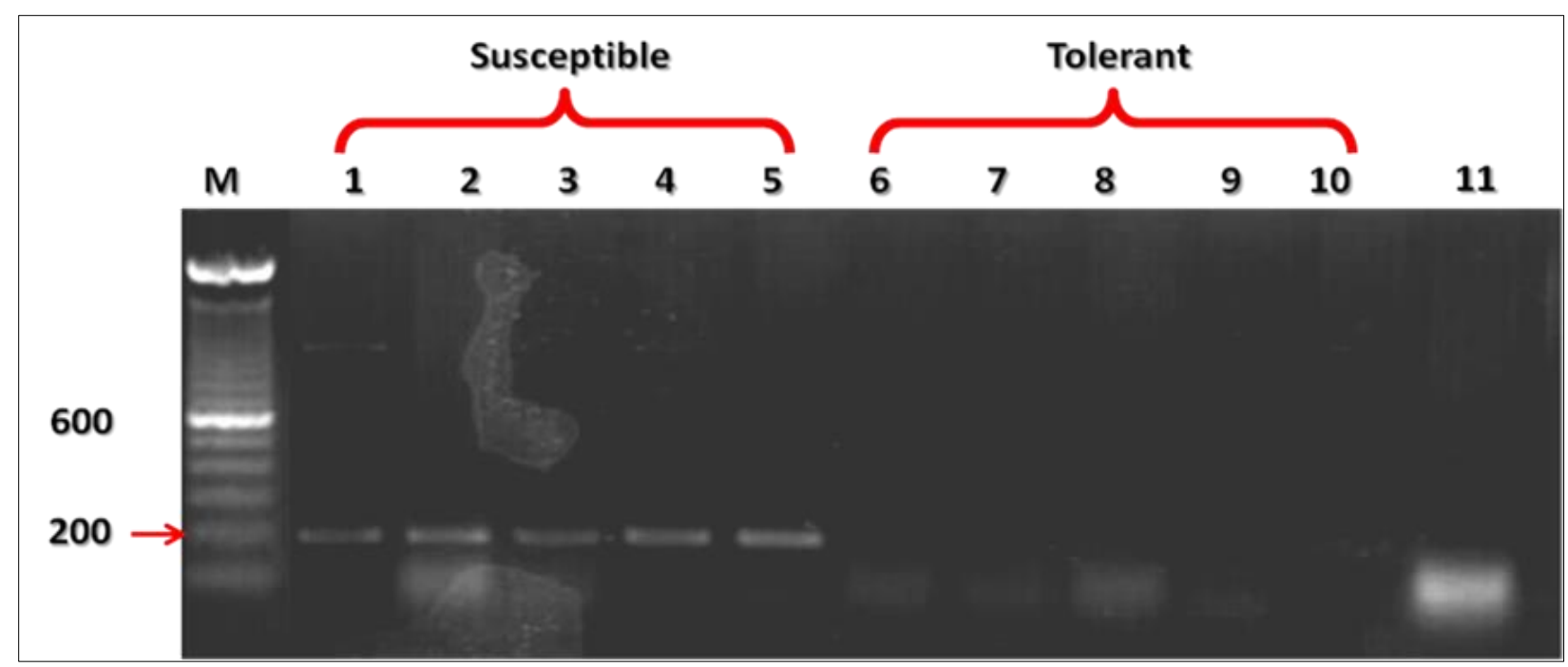

Fig 1.6: BJSCAR 1 amplification in of tolerant and sensitive cultivars. Where, 1-4 represents Susceptible genotypes namely 1 . Laxmi, 2 . IC 426392, 3. IC 399802, 4. IC 264131, and 5. Bulk of these susceptible genotypes (1-4); 6-9 represents Tolerant genotypes namely 6. IC 385686, 7. IC 312553, 8. IC 491089, 9. IC 312545, and 10. Bulk of these tolerant genotypes (6-9); 11. B. fruiticulosa (known for aphid tolerance). M indicates 100 bp DNA ladder

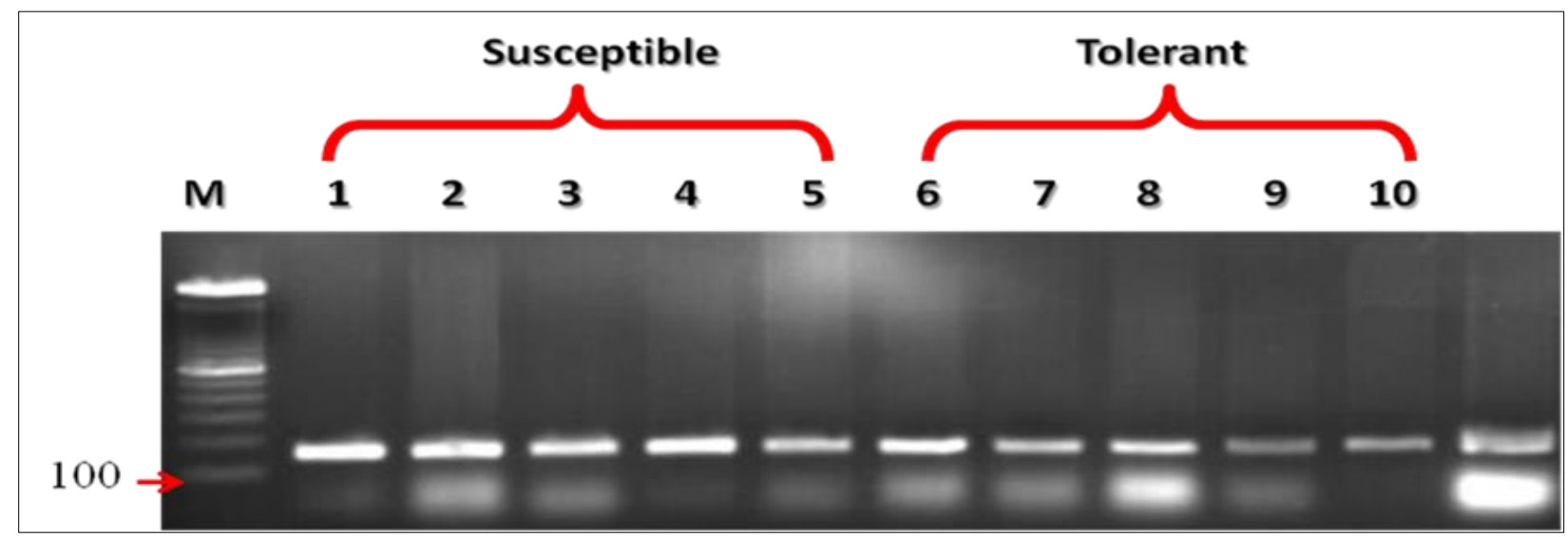

Fig 1.7: BJSCAR 2 amplification in of tolerant and sensitive cultivars. Where, 1-4 represents Susceptible genotypes namely 1 . Laxmi, 2. IC 426392, 3. IC 399802, 4. IC 264131, and 5. Bulk of these susceptible genotypes (1-4); 6-9 represents Tolerant genotypes namely 6. IC 385686, 7. IC 312553, 8. IC 491089, 9. IC 312545, and 10. Bulk of these tolerant genotypes (6-9); 11. B. fruiticulosa (known for aphid tolerance). M indicates 100 bp DNA ladder 


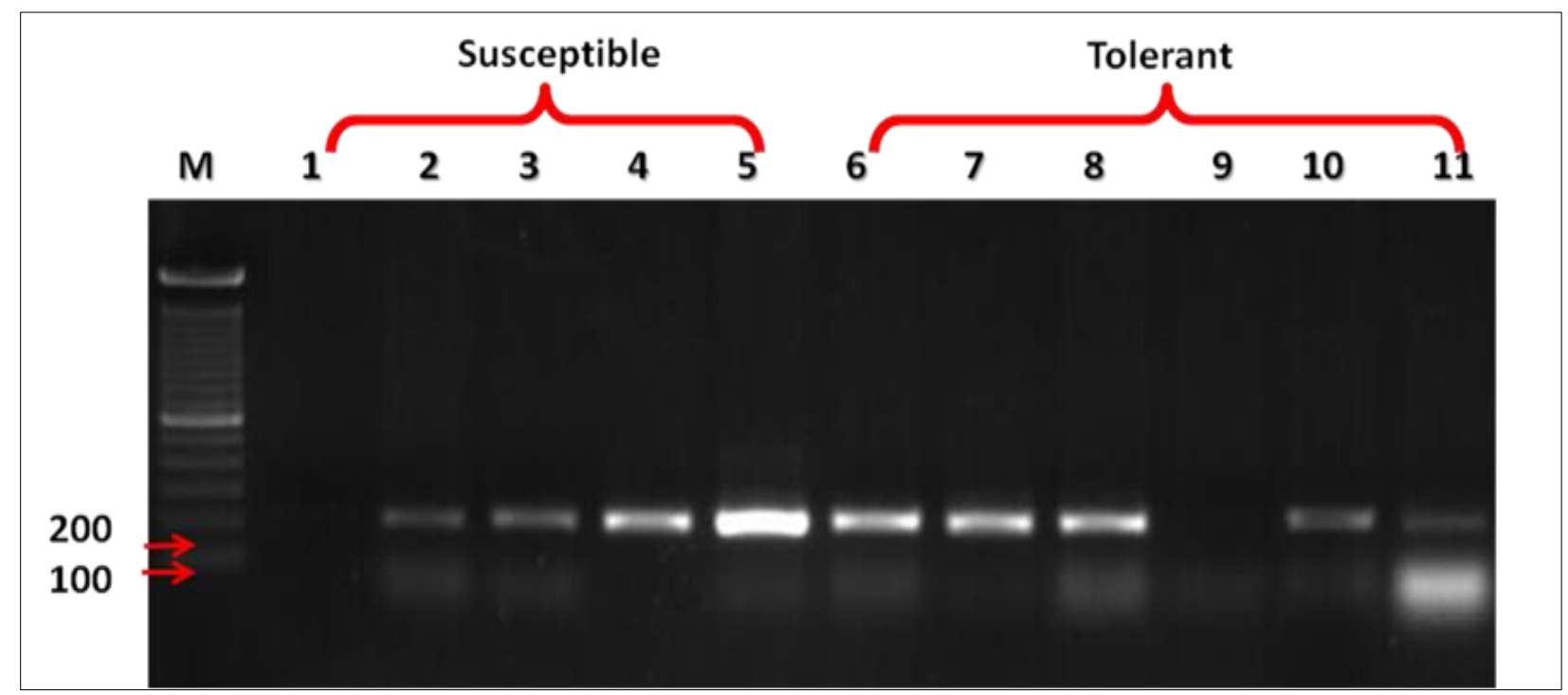

Fig 1.8: BJSCAR 3 amplification in of tolerant and sensitive cultivars. Where, 1-4 represents Susceptible genotypes namely 1 . Laxmi, 2. IC 426392, 3. IC 399802, 4. IC 264131, and 5. Bulk of these susceptible genotypes (1-4); 6-9 represents Tolerant genotypes namely 6. IC 385686, 7. IC 312553, 8. IC 491089, 9. IC 312545, and 10. Bulk of these tolerant genotypes (6-9); 11. B. fruiticulosa (known for aphid tolerance). M indicates 100 bp DNA ladder

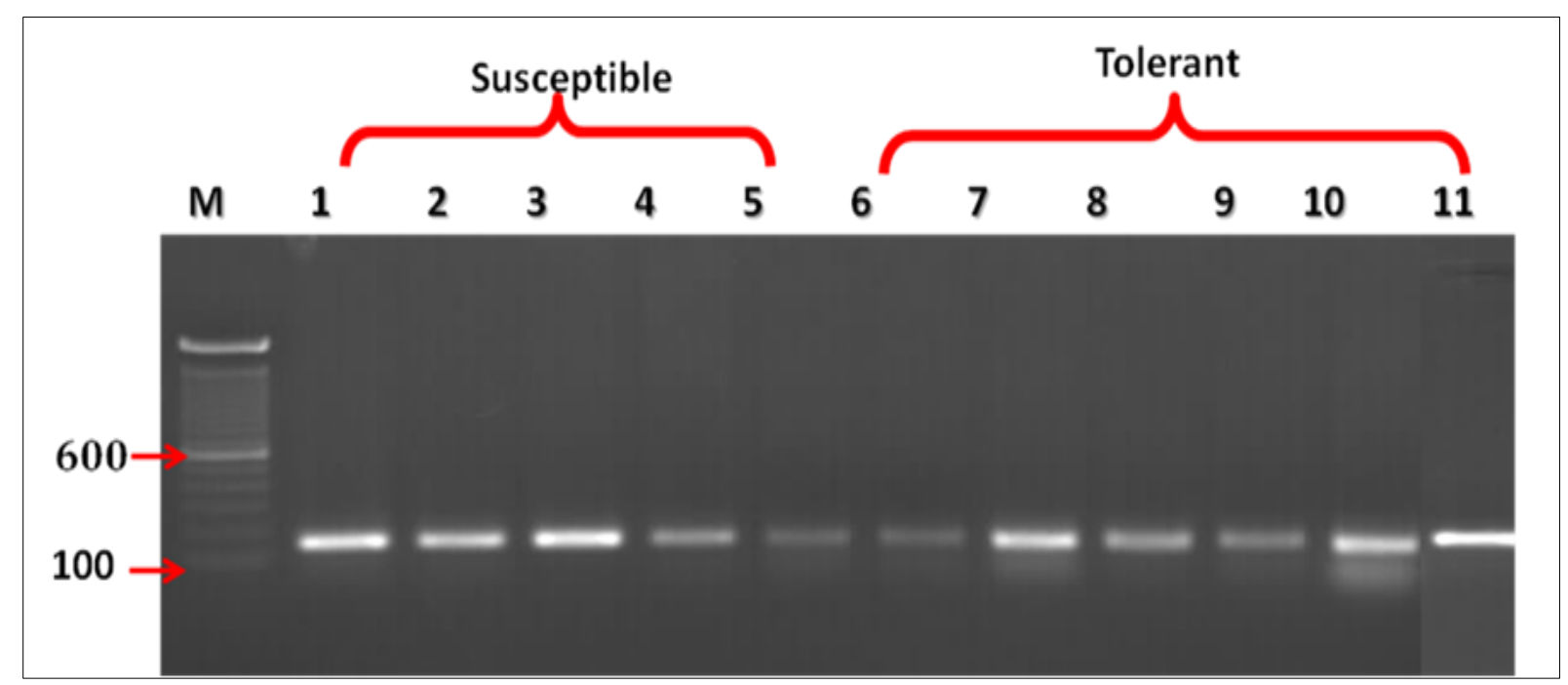

Fig 1.9: BJSCAR 4 amplification in of tolerant and sensitive cultivars. Where, 1-4 represents Susceptible genotypes namely 1 . Laxmi, 2. IC 426392, 3. IC 399802, 4. IC 264131, and 5. Bulk of these susceptible genotypes (1-4); 6-9 represents Tolerant genotypes namely 6. IC 385686, 7. IC 312553 , 8. IC 491089, 9. IC 312545, and 10. Bulk of these tolerant genotypes (6-9); 11. B. fruiticulosa (known for aphid tolerance). M indicates $100 \mathrm{bp}$ DNA ladder

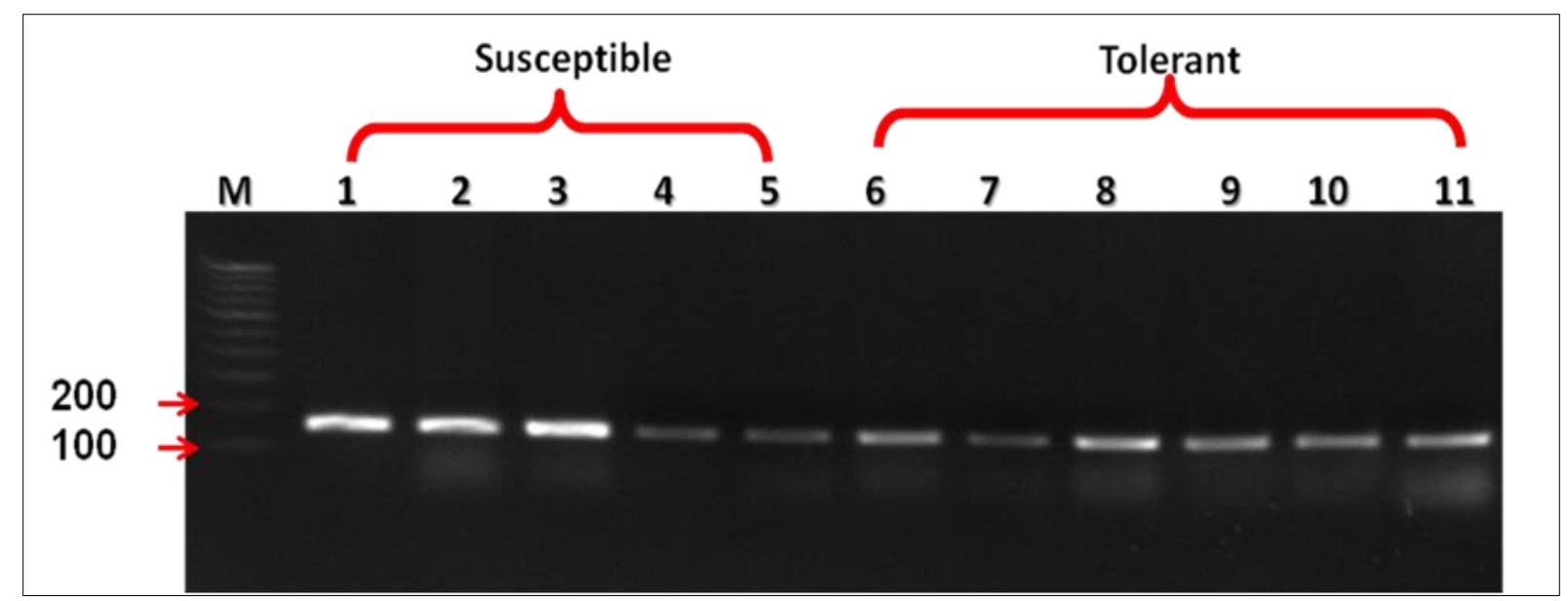

Fig 1.10: BJSCAR 5 amplification in of tolerant and sensitive cultivars. Where, 1-4 represents Susceptible genotypes namely 1. Laxmi, 2. IC 426392, 3. IC 399802, 4. IC 264131, and 5. Bulk of these susceptible genotypes (1-4); 6-9 represents Tolerant genotypes namely 6. IC 385686, 7. IC 312553, 8. IC 491089, 9. IC 312545, and 10. Bulk of these tolerant genotypes (6-9); 11. B. fruiticulosa (known for aphid tolerance). M indicates 100 bp DNA ladder. 


\section{Acknowledgement}

Authors thank Bihar Agricultural College, Sabour for all the supports and facilities for the study.

\section{References}

1. Ahuja I, Rohloff J, Bones AM. Defence Mechanisms of Brassicaceae: implications for plant-insect interactions and potential for integrated pest management. Agronomy for Sustainable Development. 2010; 30:311-348.

2. Subhash Chander, Tyagi VC, Sharma D, Bhat KV, Bhalla S. Identification of Putative Sources of Tolerance to Aphid in Mustard and Generation of Molecular Marker for Use in Crop Improvement, Int. J Curr. Microbiol. App. Sci. 2018; 7:3086-3095.

3. Damra EM, Kasrawi M, Akash MW. Development Of Scar Marker Linked To Heat Stress Tolerance In Tomato. Proceedings of 65 th ISERD International Conference, Mecca, Saudi Arabia, 23 ${ }^{\text {rd }}-24^{\text {th }}, 2017$, ISBN: 978-9386291-92-9

4. Das T, Mondal S, Mishral DK, Bhattacharyya S. Development of SCAR marker for screening Sigatokaleafspot resistance in banana genotypes. Indian J Genet. 2016; 76:69-74.

5. Doyle JJ, Doyle JL. Isolation of plant DNA from fresh tissue, Focus. 1990; 12:13-15.

6. Jacobs JME, van Eck HJ, Horsman K, Arens PFP, Verkerk-Bakker B et al. Mapping of resistance to the potato cyst nematode Globodera rostochiensis from the wild potato species Solanum vernei, Mol Breed. 1996; 2:51-60.

7. Martínez-Ballesta, del-C M, Moreno DA, Carvajal M. The Physiological Importance of Glucosinolates on Plant Response to Abiotic Stress in Brassica. Int J Mol Sci. 2013; 14:11607-11625.

8. Martins M, Sarmento D, Oliveira MM, Batlle I, Vargas FJ. Development of SCAR-CPS markers linked to tolerance-sensitivity to Fusicoccum in almond. In: Oliveira M.M. (ed.), Cordeiro V. (ed.). XIII GREMPA Meeting on Almonds and Pistachios. Zaragoza: CIHEAM, 2005, 187-191.

9. Monika G, Mirosława S, Małgorzata K. Relevance of the MI-23 marker and the potato aphid biology as indicators of tomato plant (Solanum lycopersicum) Resistance to some pests. Vegetable Crops Research Bulletin. 2010; 72:25-33.

10. Myburg AA, Cawood M, Wingfield BD, Botha AM. Development of RAPD and SCAR markers linked to the Russian wheat aphid resistance gene Dn2 in wheat, Theor Appl Genet. 1997; 96:1162-1169.

11. Paran I, Michelmore RW. Development of reliable PCR based markers linked to downy mildew resistance genes in lettuce, Theor Appl Genet. 1993; 85:985-993.

12. Prabhu KV, Somers DJ, Rakow G, Gugel RK. Molecular markers linked to white rust resistance in mustard Brassica juncea, Theor Appl Genet. 1998; 97:865-870.

13. Rai B, Sehgal VK. Field resistance of Brassica germplasm to mustard aphid Lipaphis erysimi (Kalt) Science and Culture. 1975; 41:444-445.

14. Sambrook J, Russell DW. Molecular cloning - A laboratory manual. Cold Spring Harbour Laboratory, New York, USA, 2001.

15. Srivastava MK, Li CN, Li YR. Development of sequence characterized amplified region (SCAR) marker for identifying drought tolerant sugarcane genotypes. Aust J Crop Sci. 2012; 6:763-767.
16. Srivastava RK, Mishra SK, Singh AK, Mohapatra T. Development of a coupling-phase SCAR marker linked to the powdery mildew resistance gene 'erl' in pea (Pisum sativum L.), Euphytica. 2012; 186:855-866

17. Tacconi G, Cattivelli L, Faccini N, Pecchioni N, Stanca AM et al. Identification and mapping of a new leaf stripe resistance gene in barley (Hordeum vulgare L.). Theor Appl Genet. 2001; 102:1286-1291. 